\title{
La informalidad del límite urbano: Pinares de Oriente, procesos resilientes en Medellín.
}

\author{
Inés Aquilué Junyent \\ Departament d'Urbanisme i Ordenació del Territori, \\ Universitat Politècnica de Catalunya, Barcelona, España \\ E-mail: Ines.aquilue@upc.edu
}

\begin{abstract}
Resumen. Este artículo recoge el análisis evolutivo del barrio informal de Pinares de Oriente en la ciudad colombiana de Medellín, para sentar las bases de la intervención y mejora de la seguridad ambiental del asentamiento, evitando los riesgos provocados por su implantación informal, pero sin perjudicar las relaciones socioeconómicas ya establecidas.

El sector inició su construcción producto de los procesos migratorios provocados por el conflicto armado en Colombia, iniciado en los años 1960 y con antecedentes desde el año 1948. Este asentamientos irregular, implementado desde el año 2000, fue producto de la movilidad poblacional desde ámbitos rurales, forzada por la violencia sociopolitica. Este éxodo ha provocado patrones de extensión urbana basados en la autoconstrucción, la informalidad y la ilegalidad. La situación de emergencia de Pinares de Oriente, debido a su emplazamiento inestable y su déficit en servicios y accesibilidad, requiere una intervención analítica y crítica de sus espacios relacionales. Su desarrollo urbano inicialmente ilegal, y en la actualidad, sólo parcialmente legalizado, ha alcanzado un punto crítico, puesto que el Plan de Ordenamiento Territorial (POT) de la ciudad de Medellín plantea la eliminación parcial de algunas áreas edificadas que se encuentran al límite de la zona urbana y en zonas de alto riesgo.

Se realiza un análisis espacial, que permite especificar el modelo de desarrollo seguido y que incorpora las bases históricas a considerar en las intervenciones futuras a través de los relatos en primera persona de su comunidad. Este análisis es la base para el futuro desarrollo del barrio, teniendo en cuenta la producción de su espacio y el tejido social a él vinculado. Se fija la situación espacial, concretando usos sociales y económicos, todo ello desarrollado con la participación de la comunidad, para planificar las acciones en un barrio al límite entre lo urbano y lo rural.
\end{abstract}

Palabras clave: barrios informales, desarrollo urbano-rural, morfología urbana, desplazamiento forzado, resiliencia urbana.

\section{Introducción}

La ciudad de Medellín ha sufrido grandes cambios en las últimas décadas, su población se ha multiplicado a causa de los grandes desplazamientos forzados que ha provocado la llegada de miles de personas desde zonas rurales de Antioquia. Pinares de Oriente es uno de los barrios de invasión más recientes de la ciudad, y se sitúa en el límite con el ámbito rural. Su población es prácticamente en su totalidad desplazada, y habita entre la ilusión del desarrollo sostenible y la amenaza del desalojo y de los deslizamientos de tierra.

\section{Objetivos}

El primer objetivo de la comunicación 
es contextualizar y analizar las múltiples incertidumbres que acechan una comunidad de población desplazada por el conflicto sociopolítico en Colombia. La inestabilidad política ha derivado en un estado de fuerte inestabilidad socioeconómica que a su vez se ha visto agravado por una situación espacial incierta. El artículo pretende apuntar qué factores espaciales mantienen el barrio de Pinares de Oriente en condiciones de constante incertidumbre, dificultando el desarrollo sostenible de la comunidad. Para ello, se explica que implicación tiene la aplicación del Plan de Ordenación de Medellín sobre el barrio de Pinares de Oriente y su situación de borde urbano-rural y se exponen los principales riesgos y amenazas físico-ambientales que acechan el asentamiento.

Elsegundo objetivo es mostrarqué estrategias de resiliencia promueven la estabilización del barrio para evitar un nuevo desplazamiento de la población. Para ello, se presenta la propuesta de mejoramiento integral de barrios de la Comuna 8 , que promueve la estabilización de los asentamientos física, económica, social, cultural y medioambientalmente en su ubicación existente, reforzando los lazos entre comunidad y entorno construido.

El tercer objetivo es mostrar que dadas las dificultades de producción socio-espacial de las comunidades desplazadas es necesario tejer redes complejas de ayuda mutua para hacer frente a la incertidumbre socioeconómica y espacial.

\section{Contexto urbano: La ciudad de Medellín}

La ciudad de Medellín se localiza en el Valle de Aburrá con una clara orientación nortesur, donde la vegetación y las construcciones naranjas se fusionan en un entramado denso de texturas. En el valle surcado por el río Medellín (anteriormente río Aburrá, de los indígenas Aburráes), desembocan diversas quebradas ${ }^{1}$ cuyos nacimientos se localizan en las cimas de los cerros. El primer asentamiento colonial de Medellín, cuya fundación fecha de 1616, tuvo lugar en el área centro oriental del valle junto a una de sus principales quebradas, la de Santa Elena. La cuadrícula colonial se implementó en un llano al sur de la quebrada antes de su desembocadura en el río Medellín.

Las primeras expansiones masivas de la ciudad se produjeron en el lado oriental, a modo de cuadrícula hacia el norte, donde gran parte de la población atraída por la industrialización ocupó barrios de nueva planta como Manrique y Aranjuez. Estas áreas, fueron trazadas siguiendo una cuadrícula similar a la del área central con escasa relación con la topografía de las laderas nuevamente edificadas del Valle. En el año 1938 se inició el proyecto del ensanche conocido como Otrabanda en el área occidental del valle, con los barrios de Laureles, Libertadores y la Universidad Pontificia Bolivariana. El proyecto de esta zona fue realizado por Pedro Nel Gómez, Horacio Longas y Luis de Greiff, y se componía de un plan de treinta manzanas y quinientas parcelas para casas unifamiliares en jardines. Los proyectistas propusieron una nueva forma que rompía la malla cuadrangular e incorporaba avenidas semicirculares arboladas y zonas ajardinadas (Alcaldía de Medellín 2011).

La ciudad formal se extendió en el territorio llano con cierta diferenciación en su carácter socioeconómico. Así, tradicionalmente, la zona del sur se mantuvo como área de clase pudiente y hacia el norte se situó la clase obrera. A pesar de ello, fueron las áreas de suelo no urbano, en localizaciones de pendientes extremadamente elevadas o en los lechos de las quebradas donde construyeron su hábitat las grandes masas de población desplazada forzadamente.

La ocupación periurbana de la ciudad se inició en la zona centro oriental en los siglos XVIII y XIX, con la parcelación de fincas ricas en abastecimiento de agua. Estos crecimientos de las zonas más rurales de las laderas se intensificaron a partir de los años 1950, cuando empezaron a aparecer extensos crecimientos informales coincidiendo con el inicio de La Violencia (1948-1958)2. A partir de mediados del siglo XX, las fuertes oleadas migratorias provenientes del campo a causa de las oportunidades de la urbe y las difíciles condiciones en el ámbito rural (tanto a causa del conflicto político como de las dificultades económicas) cristalizaron en la construcción de espacios de hábitat informal.

Los desplazamientos masivos, provocaron transformaciones no reguladas: la urbanización 


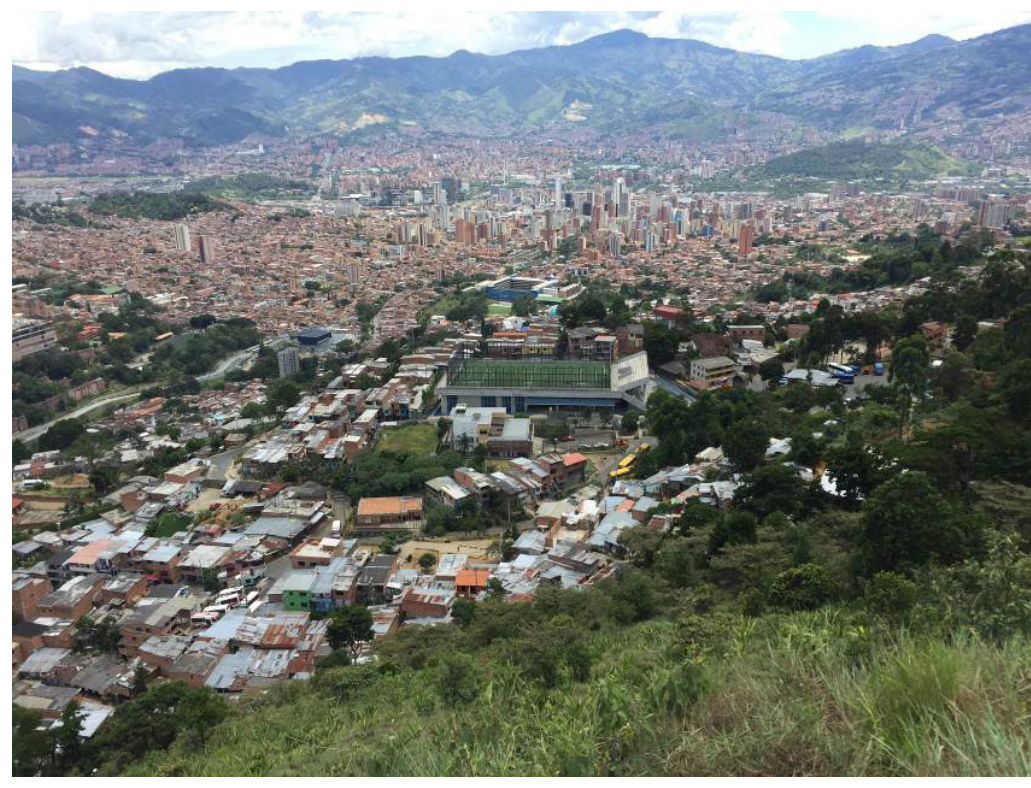

Fig. 1. Imagen desde el Cerro Pan de Azúcar. Al pie del Cerro, Pinares de Oriente, al fondo la ciudad de Medellín en el Valle de Aburrá. Fuente: Aquilué (ed.) 2018, p. 12. de áreas agrícolas, la ocupación y construcción ilegal de tierras (barrios enteros), y el asentamiento de vivienda en áreas inadecuadas, peligrosas y sin servicios, todo ello con sus consecuentes impacto ambiental y segregación poblacional. Gran parte de las áreas autoconstruidas se localizaron en las laderas de los cerros y las cuencas de las quebradas, y si bien en algunos de estos asentamientos informales se implementaron los servicios y la legalización de los predios, en otros se mantuvo la ilegalidad y la informalidad.

\section{Pinares de Oriente, el barrio}

Pinares de Oriente es un barrio pequeño del extremo centro oriental de Medellín, se sitúa en el límite de la Comuna 8, colindante al corregimiento de Santa Elena. Su comunidad es de aproximadamente 210 familias y se sitúa en la Comuna 8 (Zona Centro Oriental) de las 16 que dividen el municipio. El barrio se ubica en el límite rural de la parte alta de la comuna, en la vertiente norte del Cerro Pan de Azúcar, uno de los siete Cerros tutelares de Medellín. Adyacente a Pinares de Oriente se encuentran al sur Villatina, Villatina La Piedra y el Campo Santo; al oriente el Trece de Noviembre; y al occidente el barrio de La Libertad. El sector Pinares de Oriente consta de un área de $13.040 \mathrm{~m} 2$, y según un estudio realizado en 2014, el asentamiento disponía de 170 viviendas y unos 700 habitantes (Universidad Pontificia Bolivariana 2014), mientras que en la actualidad se sospesa que asciende a 210 viviendas.

El asentamiento fecha del año 2000, cuya población, en un $90 \%$ víctima del conflicto ${ }^{3}$, proviene de diversas zonas afectadas por desplazamientos forzados, especialmente del Oriente Antioqueño y Urabá. Tras su fundación y durante los siguientes dos años se produjeron cuatro desalojos, y la población siempre volvió a asentarse en el barrio. En el año 2005 se ordenó el último desalojo que nunca se formalizó. Así, a partir del año 2002 y hasta la actualidad, el barrio se ha asentado informalmente en un área de propiedad pública sin parcelas delimitadas administrativamente y sin ninguna titularidad. La comunidad de Pinares de Oriente es una población vulnerable en proceso de estabilización. Las reivindicaciones de mejora de la comunidad se inscriben en las demandas de la Comuna 8. Algunas de las viviendas aparecen ya construidas con materiales (ladrillo, hormigón en masa, mortero, etc.) otras se mantienen como ranchos (tablas de madera), conformando un entramado irregular de edificaciones que se arrapan a la loma del cerro, y entre las que discurren escuálidas escaleras pavimentadas con hormigón. 


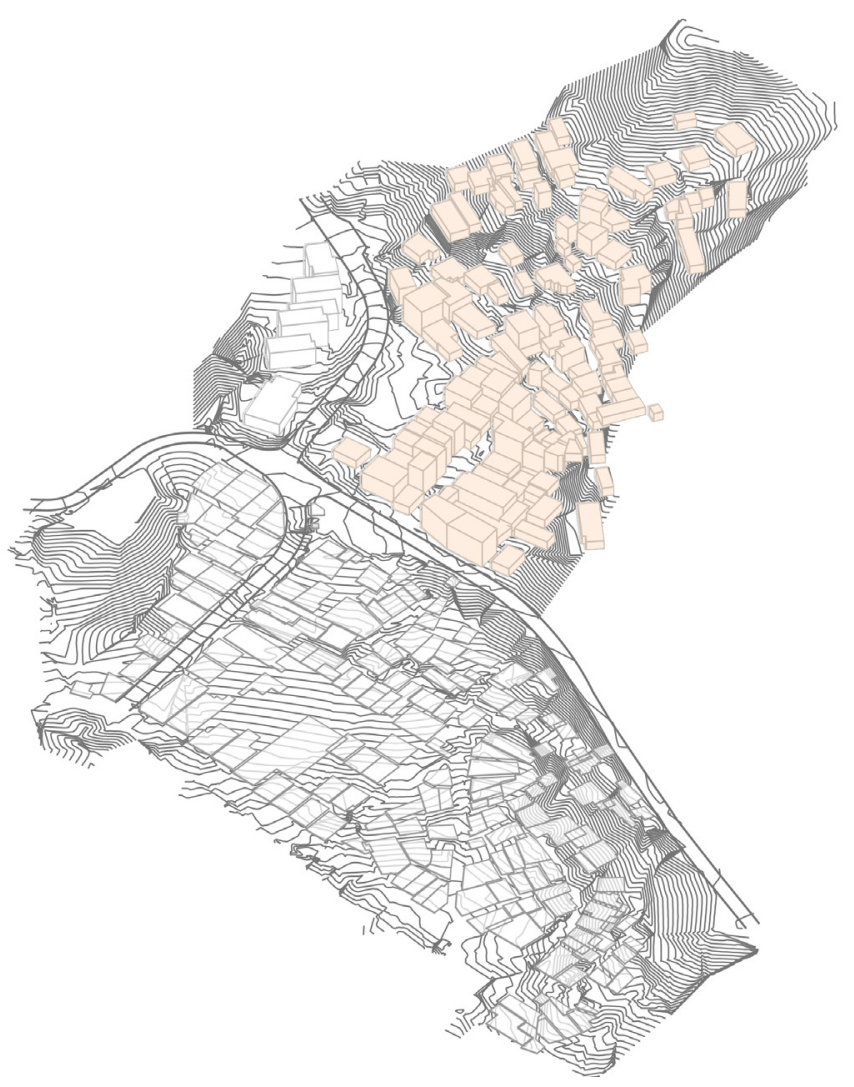

Fig. 2. Axonometría del barrio de Pinares de Oriente, donde se puede percibir la orografía del ámbito y la ocupación de la ladera por parte de los desplazados internos. Fuente: elaboración propia.

\section{Pinares de Oriente y el Plan de Ordenamiento Territorial (POT)}

Una de las mayores problemáticas que presenta el asentamiento de Pinares de Oriente era su implantación en la ladera norte del Cerro Pan de Azúcar. Por un lado existía la inseguridad legal, puesto que los suelos eran de titularidad pública, por otro lado el barrio sufría inseguridad física y estructural. Según el Informe de amenaza, vulnerabilidad y riesgo por movimientos en masa, inundaciones y avenidas torrenciales de la Empresa de Desarrollo Urbano (2016), el asentamiento de Pinares de Oriente requería ser intervenido para poder garantizar su seguridad estructural. El ámbito de Pinares con mayor riesgo de movimiento en masa y estructural es la zona con mayor pendiente del barrio, tal y como puede comprobarse en la figura 3. Los riesgos físicos condicionan la perspectiva de mejora de la Comunidad, puesto que es necesario mitigarlos.

En el último Plan de Ordenamiento Territorial (POT) de Medellín aprobado en 2014 se clasificaron las áreas de la ciudad.
Hasta el momento, el área que ocupa Pinares de Oriente había sido siempre considerada suelo rural $^{4}$, por lo que las edificaciones se encontraban fuera de ordenación. Con la modificación de 2014 la mitad de las edificaciones del barrio pasaron a ser suelo de expansión urbana. Con ello se generaba un nuevo estado para la comunidad de Pinares de Oriente, puesto que aunque seguían ocupando suelo público, la mitad del asentamiento parecía poder mantenerse mientras la otra mitad no. El presente análisis considera que esta posible nueva delimitación se debe en primer lugar a que los suelos de la parte alta tienen mayor pendiente y presentan mayor riesgo de movimiento en masa y estructural y en segundo lugar, a que la Empresa de Desarrollo Urbano (EDU) pretende reubicar, edificar y densificar en la parte baja del barrio.

En la figura 4 podemos ver la limitación de la ciudad con los cerros del Valle de Aburrá. Entre la línea discontinua y la línea continua se sitúa Pinares de Oriente, la discontinua marca el límite rural del POT de 2006, y la fina línea de puntos el límite rural del POT de 2014, partiendo el barrio en dos mitades. A pesar de 


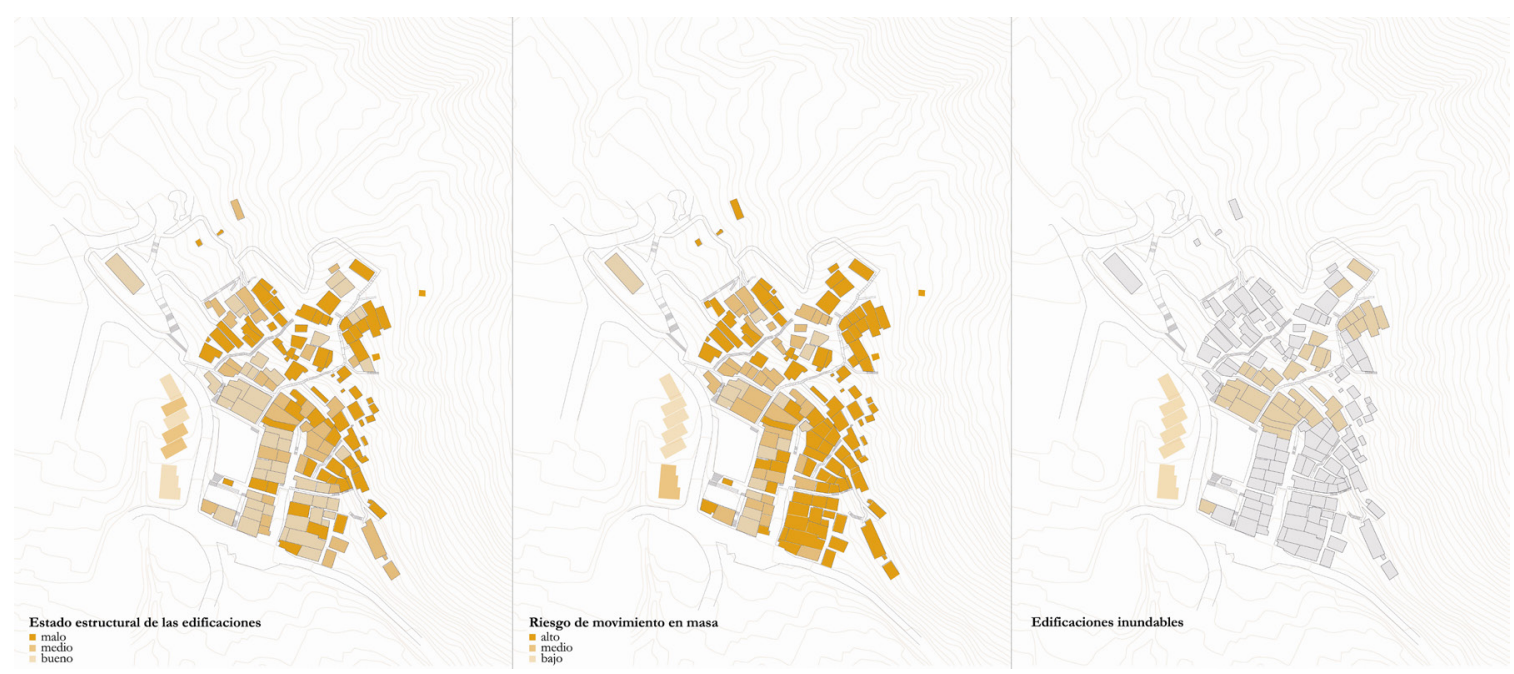

Fig. 3. Estado estructural de las edificaciones, riesgo de movimiento en masa y edificaciones inundables. Fuente: EDU 2016.

la inseguridad legal, la proximidad al Cerro de la comunidad ha permitido establecer un vínculo de retorno al desarrollo rural.

\section{Mejoramiento integral de barrios, la iniciativa}

La proliferación masiva de construcciones informales en las laderas de los cerros fue una problemáticadesatendidadurantemuchotiempo por la administración de Medellín. A partir de los años 1980 y 1990, cuando el fenómeno fue en aumento y también la violencia en la ciudad, se empezó a sospesar la intervención de la administración en zonas de alta informalidad a través de programas de mejoramiento integral de barrios, que requería comprender los procesos de construcción informales para poder intervenirlos flexiblemente. En los años 1990 se creó el PRIMED (Programa Integral de Mejoramiento de Barrios Subnormales en Medellín), que tuvo una duración de siete años y abordó la marginalidad a partir de una gestión institucional y política (Velásquez 2012). En los años 2000, el urbanismo social fue gestionado por la Empresa de Desarrollo Urbano (EDU) que focalizó sus esfuerzos en generar espacios de gran calidad y foco de actividades. Algunos de los desarrollos supusieron la construcción del metrocable en la ciudad o de los parques bibliotecas, dotando de nuevas conectividades algunas de las áreas más segregadas. En estos grandes proyectos la participación de la comunidad quedó relegada a un segundo plano, dada su relevancia infraestructural. En los últimos años la EDU ha retomado la voluntad de aplicar el mejoramiento integral, focalizándose en la mejora de la calidad de la vivienda y los espacios públicos barriales.

En el POT de 2014, el artículo 156 especifica que «la intervención de mejoramiento estará dirigida específicamente a superar las carencias de dotación del entorno: espacio público, vías, transporte $\mathrm{y}$ equipamiento social y productivo, así como también a la legalización integral de predios y edificaciones y el mejoramiento de la vivienda» y a «mejorar la mala calidad y deficientes condiciones de vida en asentamientos humanos de desarrollo incompleto e inadecuado, localizados en la periferia, los alrededores del centro de ciudad, $\mathrm{y}$ en zonas de riesgo mitigable» (POT 2014).

El mejoramiento integral de barrios se basa en la transformación social a través de una perspectiva multisectorial, que no solo interviene en el medio físico, sino que enlaza la intervención en el medio con el desarrollo social, cultural y económico de su población. En algunas ocasiones, se ha aplicado bajo las premisas del determinismo espacial, es decir, considerando, que la intervención en el espacio conducirá a la transformación social. Sin embargo, la esencia de la propuesta es el trabajo en ejes paralelos y transversales que 


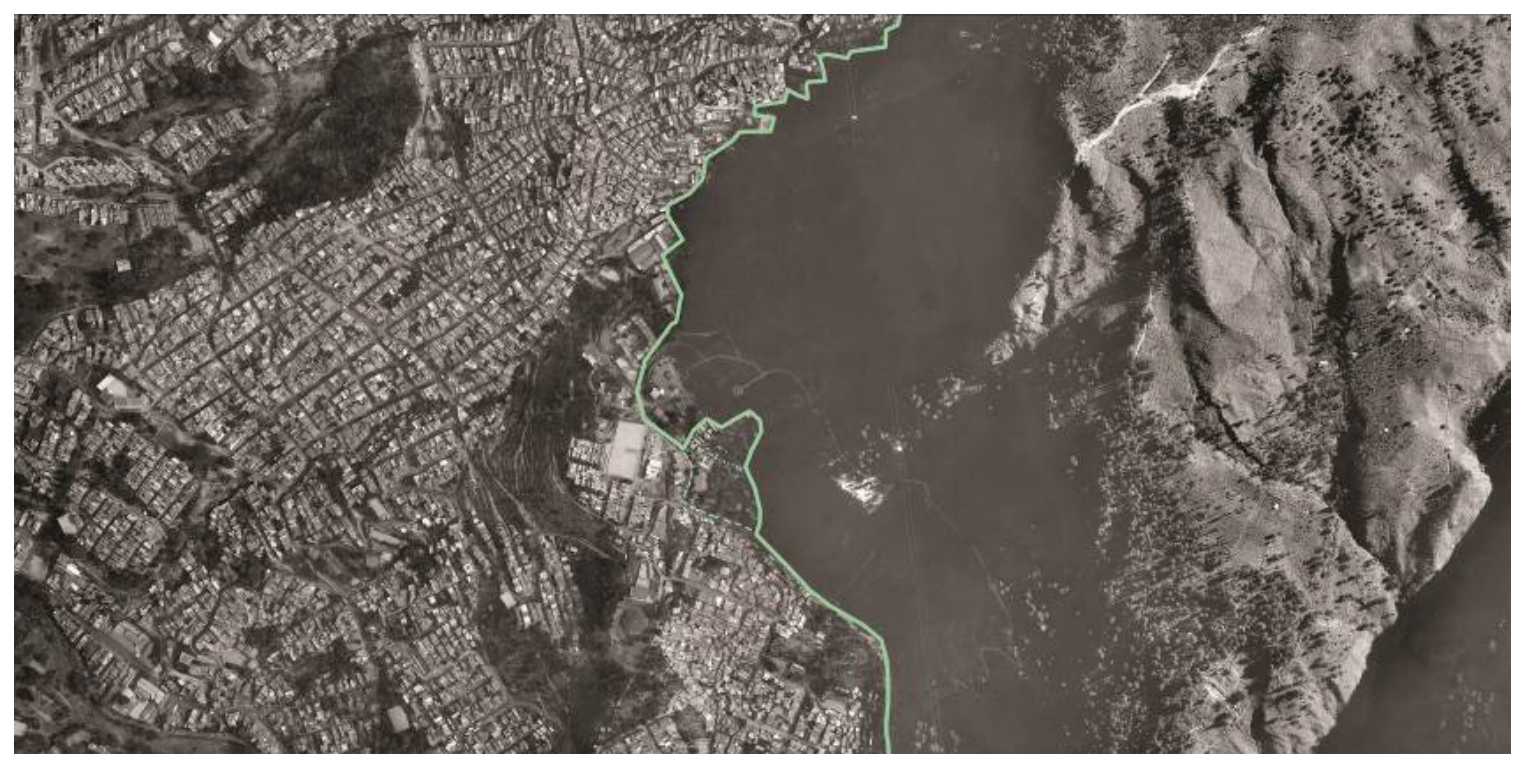

Fig. 4. Imagen aérea de la Comuna 8, límite con la zona no edificada. Fuente: elaboración propia.

relacionen la intervención espacial con las mejoras sociales, culturales y económicas. En vista de la vulnerabilidad de las zonas de acción no es suficiente acondicionar espacios públicos, sino que es necesario prever como estas intervenciones mejoran la seguridad social, económica y alimentaria de su población.

En el caso de Pinares de Oriente, dada la alta vulnerabilidad de la comunidad (casi la totalidad es víctima del desplazamiento forzado), la mejora integral supone una posibilidad de dignificar su hábitat y otorgar seguridad a sus hogares. Esta voluntad se enmarca dentro del trabajo conjunto de la comuna. El 13 de marzo de 2016 se realizó una consulta popular en toda la Comuna 8 (casi 130.000 habitantes de estratos 1, 2 y $3)^{5}$ en la que las organizaciones comunitarias refrendaron su propuesta de mejoramiento integral de barrios (Mesa de Vivienda Comuna 8, 2016). Tres eran los objetivos:

[1] Transformar las condiciones de habitabilidad de las comunidades, mejorando

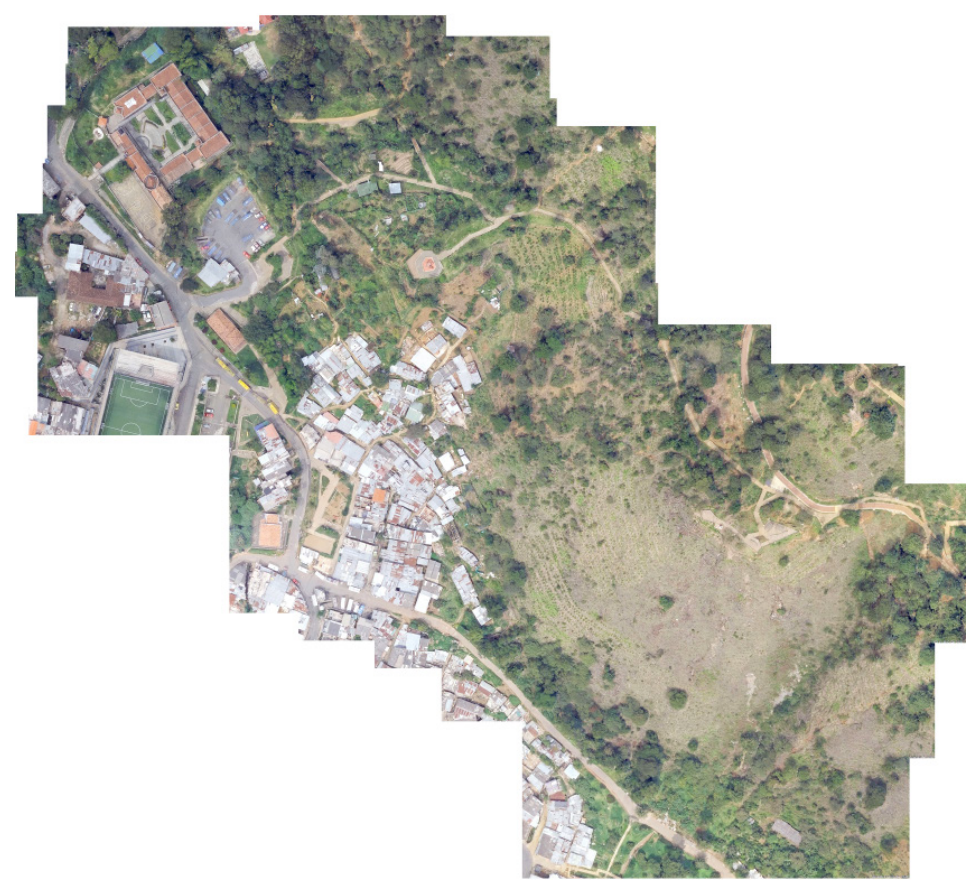

Fig. 5. Imagen aérea de Pinares de Oriente, en el Cerro Pan de Azúcar. Fuente: elaboración propia. 
Fig. 6. Imagen del Parque de Pinares de Oriente. Fuente: elaboración propia.

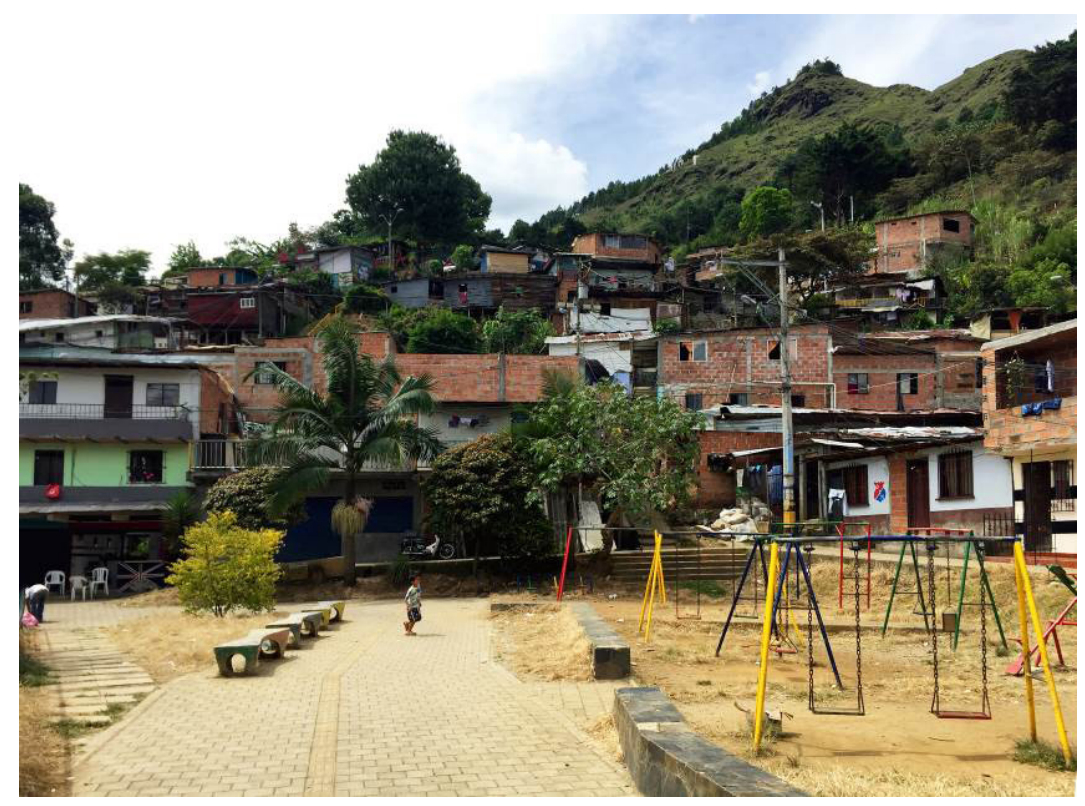

y legalizando sus viviendas y generando proyectos de viviendas nuevas acorde a las necesidades, cultura, arraigo y formas de vida en el territorio.

[2] Propiciar el equipamiento de un entorno comunitario justo que posibilite la identificación cultural y el empoderamiento político, el acceso a salud y educación integral, así como la mitigación a los riesgos estructurales o ambientales.

[3] Restablecer derechos socioeconómicos generando alternativas de empleo digno, seguridad alimentaria, formación cultural y social, y atención enfocada a las necesidades particulares de las comunidades víctimas y vulnerables.

\section{Conclusión}

El análisis físico y estructural de Pinares de Oriente ha permitido acercarnos a su compleja situación, donde el control sobre el entorno es arduo y costoso. Frente a la voluntad por eliminar parcialmente el barrio, debe considerarse que las relaciones establecidas entre la comunidad son una constante que puede evitar un nuevo desplazamiento, que tendría un alto coste social y personal, y quizás volver a apostar por el mejoramiento integral de carácter comunitario.

En Pinares, las amenazas naturales se superponen: riesgo de deslizamiento, de inundación, de avalancha, de movimiento en masa y de erosión. El emplazamiento no es seguro. Tampoco es certera la titularidad de los predios, no existe parcelación, ni títulos de las parcelas, ni registro administrativo. La propiedad es incierta.

La violencia ha provocado un entorno urbano específico: los barrios informales construidos por el desplazamiento. Las comunidades luchan por una supervivencia diaria en una situación que no se estabiliza solo con el desplazamiento. Las inseguridades han mutado, pero requieren de un fuerte esfuerzo para la supervivencia.

En Pinares de Oriente el control sobre el entorno es arduo y costoso, sin embargo, construir complejidades tejidas a través de la ayuda mutua es posible. Y así, frente a la incertidumbre el futuro de la comunidad yace en su complejidad social, física y económica.

\section{Notas}

1 En Colombia, quebrada es un arroyo que desciende por una quiebra, sus cauces varían según las estaciones. En Medellín, existen decenas de quebradas que desembocan en el Río Medellín.

2 La Violencia (1948-1958) es un período histórico de Colombia, donde hubo enfrentamientos entre simpatizantes del 
Partido Liberal y el Partido Conservador.

3 Según las entrevistas realizadas en febrero de 2017 a miembros de la comunidad, casi el $90 \%$ de las familias son víctimas del desplazamiento forzado.

4 En el Plan de Ordenación Territorial de Medellín el suelo se clasifica en suelo rural, suelo urbano y suelo de expansión urbana (POT, 2014).

5 En Colombia, las áreas urbanas están clasificadas según la estratificación socioeconómica. Existen seis estratos: [1] Bajo-bajo; [2] Bajo; [3] Bajo-Medio; [4] Medio; [5] Medio-Alto; [6] Alto.

\section{Referencias}

Alcaldía de Medellín (ed.). 2011. Medellín: una ciudad que se piensa y se transforma. Departamento Administrativo de Planeación 50 años (1960 - 2010). Medellín: Alcaldía de Medellín.

Aquilué, Inés (ed.). 2018. Resiliencia frente a Violencia: Construcción de futuros en Pinares de Oriente. Barcelona: Iniciativa Digital Politècnica.

Busquets, Joan. 1999. La urbanización marginal. Barcelona: Edicions UPC.

Empresa de Desarrollo Urbano; Siga Ingeniería y Consultoría S.A. (2016). Informe de amenaza, vulnerabilidad y riesgo por movimientos en masa inundaciones y avenidas torrenciales. Polígono Pinares de Oriente. Medellín: EDU.

Mesa de Vivienda y Servicios Públicos Domiciliarios - Comuna 8; Mesa de Desplazados - Comuna 8; Observatorio de Seguridad Humana de Medellín; Mesa Interbarrial de Desconectad@s. 2016. Lineamientos comunitarios para una política pública de Mejoramiento Integral de Barrios. Medellín: Comuna 8 de Medellín.

Plan de Ordenación Territorial de Medellín. 2014. Acuerdo 48 de 2014: Por medio del cual se adopta la revisión y ajuste de largo plazo del Plan de Ordenamiento Territorial del Municipio de Medellín y se dictan otras disposiciones complementarias. Medellín: Alcaldía de Medellín.

Universidad Pontificia Bolivarina. 2014.
Polígono de intervención Pinares de Oriente - Villatina La Piedra: Barrios sostenibles. Documento técnico de soporte fase de diagnóstico. Medellín: EDU; Alcaldía de Medellín.

Velásquez, Carlos. 2012. "La política de Mejoramiento Integral de Barrios en Medellín. ¿Reconquista del territorio por parte del Estado?" Kavilando 3 (1-2): 72-78. 